\begin{tabular}{|c|l|}
\hline Title & An experimental comparison between KELM and CA RT for crop classification using Landsat-8 OLI data \\
\hline Author(s) & Sonobe, Rei; Tani, Hiroshi; Wang, X iufeng \\
\hline Citation & $\begin{array}{l}\text { Geocarto international, 32(2), 128 138 } \\
\text { https:/doi.org/10.1080/10106049.2015.1130085 }\end{array}$ \\
\hline Issue Date & 2016 \\
\hline Doc URL & http://hdl.handle.net/2115/64502 \\
\hline Rights & $\begin{array}{l}\text { This is an Accepted Manuscript of an article published by Taylor \& Francis in Geocarto international on 12 Jan 2016, } \\
\text { available online: http:/www.tandfonline.com/10.1080/10106049.2015.1130085 }\end{array}$ \\
\hline Type & article (author version) \\
\hline File Information & Geocarto Int.32-2_128 138.pdf \\
\hline
\end{tabular}

Instructions for use 


\section{An experimental comparison between KELM and CART for crop classification using Landsat-8 OLI data}

The Operational Land Imager (OLI) is the latest instrument in the Landsat series of satellite imagery, which officially began normal operations on May 30, 2013. The OLI includes two bands that are not on the Thematic Mapper (TM) series of sensors aboard Landsat-5 and 7; a cirrus band and a coastal/aerosol band. This paper compares the classification and regression tree (CART) and the kernel-based extreme learning machine (KELM) for mapping crops in Hokkaido, Japan, using OLI data, except the cirrus band and the pan band. The OLI data acquired on July 8, 2013 was used for crop classification of beans, beets, grassland, maize, potatoes, and winter wheat. The KELM algorithm performed better in this study and achieved overall accuracies of $90.1 \%$. According to the Jeffries-Matusita (J-M) distances, the short wavelength infrared band provides the greater contribution (the highest value was observed for band 6 in OLI data).

Keywords: classification and regression tree; kernel-based extreme learning machine; Landsat-8; operational land imager.

\section{Introduction}

Land-cover classification is one of the most common applications of remote sensing. Crop classification maps are useful for estimating yield amount or the acreage of tillable land and managing the agricultural field. Satellite data have been tested with variable results, both optical (Hartfield et al. 2013; Sarker and Nichol 2011; Sonobe et al. 2014a) and SAR data (Laurin et al. 2013; Sonobe et al. 2014bc). Optical remote sensing is also one of the most attractive options for obtaining biomass information and forest monitoring. The sensors suitable for identifying species or conditions of vegetation are Landsat series (Ding et al. 2014; Goodwin and Collett 2014; Hartfield et al. 2013), SPOT series (Cockx et al. 2014; Langner et al. 2014; Yuan et al. 2014), and ALOS/AVNIR-2 (Sarker and Nichol 2011). 
Classification algorithms are essential for the accuracy of the generated map, which shows the crop status. A number of approaches, including statistical and machine learning algorithms, have been developed and employed for land cover classification within the past 30 years. Machine learning approaches have been widely used in image classification over the past two decades (Pal et al. 2013). In earlier studies, the classification and regression tree (CART) algorithm was used to identify crops such as alfalfa, corn, cotton, grain, melon orchards, and sorghum from Landsat TM image data (Hartfield et al. 2013). Recently, a new fast learning neural algorithm for single hidden layer feedforward neural networks (SLFNs), named the extreme learning machine (ELM), was developed to improve the efficiency of SLFNs. ELM has been successfully applied to many real-world applications, such as classification and regression problems (Huang et al. 2012).

The main objectives of this work are to (1) evaluate the potential of Landsat-8 OLI images for crop type classification including the Kauth Thomas (KT) transform modified for OLI (Baig et al. 2014) and (2) compare the results of CART and KELM for the crop type classification.

\section{Materials and Methods}

\subsection{Study area}

The experimental area of this study was comprised of agricultural fields in the western Tokachi plain, Hokkaido, Japan (Figure $1,142^{\circ} 55^{\prime} 12^{\prime \prime}$ to $143^{\circ} 05^{\prime} 51^{\prime \prime}$ E, $42^{\circ}$ $52^{\prime} 48^{\prime \prime}$ to $43^{\circ} 02^{\prime} 42^{\prime \prime} \mathrm{N}$ ) at an elevation between 50 and $230 \mathrm{~m}$. The climate of the study area is characterized by warm summers and cold winters with an average 
annual temperature of $6^{\circ} \mathrm{C}$ and annual precipitation of $920 \mathrm{~mm}$.

The mean size of the fields was 2.16 ha. The dominant crops are beans (Vigna angularis and Glycine max), beets (Beta vulgaris), grassland (Phleum pratense or Dactylis glomerata), maize (Zea mays), potatoes (Solanum tuberosum), and winter wheat (Triticum aestivum). The cultivation calendar for the crops in this study area is presented in Table 1. The OLI data acquisition date (July 8, 2013) was included the growing season for the six crops, and the data may contribute to the characterization of the plant structures.

$<$ Figure 1 >

$<$ Table 1>

\subsection{Satellite data}

The Landsat-8 OLI data were observed on July 8, 2013. The data were downloaded from EarthExplorer (http://earthexplorer.usgs.gov/) and had been spatially orthorectified by USGS. The acquired scene was overlaid to verify successful orthorectification of the images. Although the OLI image includes nine bands, the pan band (band 8) and the cirrus band (band 9) were not used for classification in this study. The Fast Line-ofsight Atmospheric Analysis of Spectral Hypercubes (FLAASH) (Matthew et al. 2000) algorithm was used to correct the imagery for atmospheric effects. It was reported that Multi-date Landsat TM images can improve crop classification (Cohen and Shoshany 2002) and the 17 observations were conducted by Landsat- 8 OLI from May to August 2013 for the whole site. However, only one date (July 8) during the growing season was used because it was of better quality for classification purposes; the other images were covered with cloud making it difficult to distinguish crop files and bare soil due to the low plant heights. 
The earlier studies show that the data of the KT transform, also known as the TasseledCap transform, are effective for improving the accuracy of the crop classification (Crist 1986; Hartfield et al. 2013). In this study, KT data were generated using the KT coefficients for OLI (Baig et al. 2014).

\subsection{GIS data}

Reference data were provided by Tokachi Nosai (located in Obihiro city, Hokkaido. Nosai conducts an Agricultural Insurance Scheme, which helps stabilize farmers suffering from damage caused by natural disasters and contributes to the growth of Japanese agriculture) as a polygon shape file, which included the position of the fields and attribute data, such as crop types. Any field below 2.0 ha was deemed too small for analysis and removed from the vector maps (Hartfield et al. 2013). The position of the center of the fields was calculated and the reflectance values of all bands were extracted using these points. The process was applied to avoid selecting training pixels from the edge of a field, which would create a mixed signal and affect the accuracy assessment.

\subsection{Separability assessment}

Before classification, J-M distances (Richards 1999) were calculated to compare statistical separability among crop types. The J-M distance is a widely used statistical separability criterion. It is a parametric criterion, for which the values range between 0 and 2. For the separability measurement, the normal distribution is usually considered. In this case, the J-M separability criterion takes into account the distance between class means and the distribution of values from the means. This is achieved by involving the covariance matrices of the classes in the separability measurement. This separability criterion can be used to pairwise measure the separability between classes, allowing the assessment of the quality of the selected class samples in the available feature space 
(Dabboor et al. 2014). As a general rule, if the J-M value is greater than 1.9, separation is good. If the J-M is between 1.7 and 1.9 , separation is fairly good.

\subsection{Classification}

We used a stratified random sampling approach to select the fields used for training, and $20 \%$ of crop fields were selected at random as training samples (Hartfield et al. 2013). The remaining $80 \%$ of the fields were used to perform the accuracy assessment. Table 2 represents the numbers of fields of each crop type.

$<$ Table 2>

The CART algorithm was popularized by Breiman et al. (1984). In CART, the Gini index is a measure of how often a randomly chosen element from the training data set would be incorrectly labeled if it were randomly labeled according to the distribution of labels.

In addition to CART, the KELM (Huang et al. 2012) was used in this study. In ELM ( Huang et al. 2004), the initial parameters of the hidden layer need not be tuned and almost all nonlinear piecewise continuous functions can be used as the hidden neurons. Therefore, $\mathrm{N}$ for arbitrary distinct samples, $\{(\mathrm{xi}, \mathrm{ti}) \mid \mathrm{xi} \in \mathrm{Rn}, \mathrm{ti} \in \mathrm{Rm}, \mathrm{i}=1, \cdots, \mathrm{N}\}$ the output function in ELM with hidden neurons is

$$
f_{L}(x)=\sum_{i=1}^{L} \beta_{i} h_{i}(x)=h(x) \beta
$$

where $\beta=\left\{\beta_{1}, \beta_{2}, \ldots, \beta_{\mathrm{L}}\right\}$ is the vector of the output weights between the hidden layer of L neurons and the output neuron, and $h(x)=\left\{h_{1}(x), h_{2}(x), \ldots, h_{L}(x)\right\}$ is the output vector of the hidden layer with respect to the input $\mathrm{x}$, which maps the data from the input space to the ELM feature space. 
For decreasing the training error and improving the generalization performance of neural networks, the training error and the output weights should be minimized at the same time; that is,

$$
\text { Minimize: }\|H \beta-T\|,\|\beta\| \text {. }
$$

The least squares solution of (2) based on Karush-Kuhn-Tucker (KKT) conditions (Fletcher 1981) can be written as

$$
\beta=H^{T}\left(\frac{1}{C}+H H^{T}\right)^{-1} T
$$

where $\mathrm{H}$ is the hidden layer output matrix, $\mathrm{C}$ is the regulation coefficient, and $\mathrm{T}$ is the expected output matrix of samples. Then, the output function of the ELM learning algorithm is

$$
f(x)=h(x) H^{T}\left(\frac{1}{C}+H H^{T}\right)^{-1} T .
$$

If the feature mapping $\mathrm{h}(\mathrm{x})$ is unknown and the kernel matrix of ELM based on Mercer's conditions can be defined as follows:

$$
M=H H^{T}: m_{i j}=h\left(x_{i}\right) h\left(x_{j}\right)=k\left(x_{i}, x_{j}\right),
$$

the output function $\mathrm{f}(\mathrm{x})$ of the KELM can be written compactly as

$$
f(x)=\left[k\left(x, x_{i}\right), \ldots, k\left(x, x_{N}\right)\right]\left(\frac{1}{c}+M\right)^{-1} T,
$$

where $\mathrm{M}=\mathrm{HH}^{\mathrm{T}}$, and $\mathrm{k}(\mathrm{x}, \mathrm{y})$ is the kernel function of hidden neurons of SLFNs. Many kernel functions satisfy the Mercer condition in existing literature, such as linear kernel, polynomial kernel, Gaussian kernel, and exponential kernel. For KELM classification, the Radial Basis Function (RBF) kernel was applied and the kernel is as follows:

$$
k(x, y)=\exp (-a\|x-y\|)
$$


Therefore, the number of kernel parameters (a) and the regularization coefficient should be tuned in order to apply the RBF kernel.

Optimal parameters for flexibility control were determined through a grid search in the bivariate parameter space. The parameter space was discretized along $2^{\mathrm{x}}$, where $\mathrm{x}=10$ to 20 for the regularization coefficient, and $\mathrm{x}=16$ to 30 for the number of kernel parameters. Both parameters were determined using the k-fold cross-validation technique. The grid search was used to minimize the misclassification error rate. K-fold cross-validation was also used to assess classifier performance (Puertas et al. 2013). This technique repeatedly generates training and test data sets from a reference sample with known land cover class membership. It is used for model validation and consists of partitioning the data into $\mathrm{k}$ equally-sized subsets (here, $\mathrm{k}=10$ ). A classifier is trained on all except one of these subsets and evaluated on the excluded subset. Accuracy measures are averaged over all test datasets. KELM was applied using MATLAB and Statistics Toolbox Release 2014b (The MathWorks, Inc., Natick, Massachusetts, United States) and the source code was downloaded from http://www.ntu.edu.sg/home/egbhuang/index.html. CART was applied using R version 3.0.0 (RCoreTeam 2013) and 'rpart' package (Therneau et al. 2013).

We conducted classifications using the following three different datasets: (1) reflectance data, (2) KT data, and (3) reflectance + KT data. The classification maps based on the three datasets and two algorithms were evaluated in terms of their overall accuracy (OA), producer's accuracy (PA), and user's accuracy (UA). Furthermore, measures of quantity disagreement (QD) and allocation disagreement (AD) (Pontius and Millones 2011) were used for evaluation. Although the kappa statistic (Cohen 1960) had been used as an accuracy measure, it has fundamental conceptual flaws, such as being undefined even for simple cases, or having no useful 
interpretation (Pontius and Millones 2011). Therefore, QD and AD are widely used as alternative measures of the kappa statistic (Baker et al. 2013; Farewell et al. 2013; Varshney et al. 2012). QD is defined as the difference between the reference data and the classified data based upon a mismatch of class proportions. AD can be considered as the difference between the classified data and the reference data due to incorrect spatial allocations of fields in the classification.

In order to compare the accuracy of classification methods, McNemar's test (McNemar 1947) or Z-test (Congalton and Green 2008) were used. McNemar's test takes into account the use of no independent samples by focusing on how each point was either correctly or incorrectly classified in the two classifications being compared. A chisquared value of 3.84 indicates a significantly different OA between the two methods at the $95 \%$ significance level. The value of $\mathrm{Z}$-score is an approximation of the standard normal deviate of 1.96 for the $95 \%$ two-sided confidence level. Since the purpose is to reveal the best algorithm for crop type classification in this study, the Z-test was performed for a pairwise comparison of the proposed methods.

\section{Results and Discussion}

\subsection{Separability assessments}

The J-M separability measurements were computed for all obtained data (the training data and the test data) for all possible pairs of crop types (Figure 2). The short wavelength infrared bands, especially band 6, were useful. Moreover, band 5, greenness and wetness, may be effective for the separation between beans and potatoes, which is low for band 6. Values for the pairs of beans-beets, beans-wheat, beets-potatoes, grassland-wheat, and potatoes-wheat were over 1.7; whereas, values were lower than 1.0 for beans-grassland and beans-maize. 
$<$ Figure 2>

\subsection{Classification model by CART}

Figure 3 shows the classification model by CART after pruning. In cases where only the reflectance data were used (Figure 3(a)), wheat fields were only identified by the low reflectance of band 6, which may be due to the high separability measurements. For wheat, the separability measurements were over 1.7 except for the pair with maize; however, the measurement of band 6 for maize-wheat was the highest value among the reflectance and KT data. For classification, band 6 was used in four nodes, bands 3 and 5 were used in two nodes, and bands 1, 2, and 4 were not used for classification. In cases where only the KT data were used (Figure 3(b)), brightness was used in eight nodes and wetness was used in five nodes, while greenness and haziness were not used for classification. In cases where the reflectance of the bands and the KT data were used (Figure 3(c)), band 6 was used in five nodes.

$<$ Figures 3>

\subsection{Parameter tuning of KELM Classifiers}

For the application of KELM, the number of hidden nodes was tuned using the training data and the k-fold cross-validation technique. In cases where only the reflectance data were used, a higher accuracy was observed when the number of kernel parameters was $2^{27}$, the regularization coefficient was $2^{14}$ and thus, this value was adopted for KELM classification. In cases that used the KT data, a higher accuracy was observed when the number of kernel parameters was $2^{27}$ and the regularization coefficient was $2^{15}$ and thus, this value was adopted for KELM classification. In cases using the reflectance of the bands and the KT data, a higher accuracy was observed when the number of kernel 
parameters was $2^{28}$ and the regularization coefficient was $2^{13}$ and thus, this value was adopted for KELM classification.

\subsection{Accuracy Validation}

Table 3 presents the corresponding confusion matrices of classifications and Table 4 presents the Z-test results. For KELM, the classification result of dataset (1) was superior to the other combinations; however, the differences of the accuracy results are not confirmed at the $95 \%$ significance level. For CART, no differences were found between the results of dataset (1) and those of dataset (3); although the classification results of dataset (2) were inferior to those of (1) and (3). As a result, there is no advantage of adding KT data in this case.

$<$ Table 3>

$<$ Table 4>

Although the separabilities for beans-grassland and beans-maize were lower, all PA and UA were over 0.725 for KELM. Almost all PAs and UAs of KELM were superior to those of CART. The 31 maize fields were misclassified as beans and the 36 bean fields were misclassified as maize fields in CART, while the 12 maize fields were misclassified as beans and the 39 bean fields were misclassified as maize fields in KELM. Therefore, CART is superior to KELM in the PA for maize. Regarding OA, AD, and QD, the results of KELM were superior to those of CART. We used the Z-test to compare the accuracy of the classification methods because the same samples and the same assessment points were used for each classification. The difference between CART and KELM was meaningful $(p<.05)$ since the Z-score was 5.75. In the classification using KELM, there were 195 misclassified fields, consisting of 26 bean fields ( $8.1 \%$ of total beans fields in the test data), 19 beet fields (7.0\%), 52 grasslands (20.2\%), 55 maize fields (27.5\%), 20 potato fields (10.1\%), and 23 winter wheat fields 
(3.2\%). The KELM classifier particularly confused maize fields with bean fields (39 fields). The J-M distance between them ranged from 0.285 to 0.726 , and the separability was very low. Multi-temporal images can improve crop classification because they contain information on among-crop variation in growth patterns (Cohen and Shoshany 2002; Hartfield et al. 2013). In future studies, the potential of multi-temporal OLI images analyzed for crop type classification will be tested. However, applying KELM, the crop type map at the high rate of $90 \%$ was generated from only one set of OLI data. Although the conditions such as study area and crop type in this study differ from those in Hartfield et al. (2013), the OA was close to theirs, indicating 92\% accuracy using multi-temporal Landsat TM data (6 scenes).

In this study, band 6 made a significant contribution to the good results. The reflectance at shortwave infrared is sensitive to non-structural carbohydrates (NSC) of the leaf (Asner and Martin 2015), and a positive correlation is observed between leaf $\mathrm{N}$ and total shortwave reflectance (Bartlett et al. 2011). As a result, the additional use of shortwave infrared data contributed to the estimation of photosynthetic pigments, water, nitrogen, cellulose, lignin, phenols, and leaf mass per area (LMA), which accurately estimated the canopy traits (Asner et al. 2011). Furthermore, the maximum rates of carboxylation (Vcmax) and electron transport (Jmax) are estimated using two narrow bands at shortwave infrared (unpublished); thus, band 6 data may be related to the leaf photosynthetic traits. On the observation date, the differences between the photosynthetic rates of the six species were ambiguous because the potato growth was inhibited by chemicals for easy harvesting, and the growing seasons of beets, beans and maize were in the middle period but their structures were different, while the growing seasons of wheat and potato were close to the end. Thus, band 6 revealed the differences related to photosynthesis among the six species. 


\section{Conclusions}

To generate a classification map, only one image from Landsat-8 OLI was used since all images except the data acquired on July 8 were covered with clouds and could not be used for analysis, even though the 17 observations conducted by Landsat-8 OLI from May to August 2013 covered the whole site.

Of the two algorithms, CART and KELM, that were applied, the accuracy results of KELM were superior. This approach should be applicable to generate land cover/use classification maps, particularly for agricultural fields. The KELM classifier was able to generate the crop classification map of the monitored study area with an OA of $90.1 \%$. Some missions operate some satellite constellations and are capable of field based, regional or global scale agricultural monitoring on a frequent revisit cycle. The approach described in this paper will be applied by on-boarding the sensor, which can observe shortwave infrared regions.

\section{References}

Asner, G.P. and R.E. Martin. 2015. Spectroscopic remote sensing of non-structural carbohydrates in forest canopies. Remote Sensing 7, no 4: 3526-47.

Asner, G.P., R.E. Martin, D.E. Knapp, R. Tupayachi, C. Anderson, L. Carranza, P. Martinez, M. Houcheime, F. Sinca and P. Weiss. 2011. Spectroscopy of canopy chemicals in humid tropical forests. Remote Sensing of Environment 115, no 12: 3587-98.

Baig, M.H.A., L.F. Zhang, T. Shuai and Q.X. Tong. 2014. Derivation of a tasselled cap transformation based on landsat 8 at-satellite reflectance. Remote Sensing Letters 5, no 5: 423-31.

Baker, B.A., T.A. Warner, J.F. Conley and B.E. Mcneil. 2013. Does spatial resolution matter? A multi-scale comparison of object-based and pixel-based methods for detecting change associated with gas well drilling operations. International Journal of Remote Sensing 34, no 5: 1633-51.

Bartlett, M.K., S.V. Ollinger, D.Y. Hollinger, H.F. Wicklein and A.D. Richardson. 2011. Canopy-scale relationships between foliar nitrogen and albedo are not observed in leaf reflectance and transmittance within temperate deciduous tree species. BotanyBotanique 89, no 7: 491-97. 
Breiman, L., J. Friedman, C.J. Stone and R.A. Olshen. 1984. Classification and regression trees Wadsworth statistics/probability. London: Taylor \& Francis.

Cockx, K., T. Van De Voorde and F. Canters. 2014. Quantifying uncertainty in remote sensingbased urban land-use mapping. International Journal of Applied Earth Observation and Geoinformation 31: 154-66.

Cohen, J. 1960. A coefficient of agreement for nominal scales. Educational and Psychological Measurement 20, no 1: 37-46.

Cohen, Y. and M. Shoshany. 2002. A national knowledge-based crop recognition in mediterranean environment. International Journal of Applied Earth Observation and Geoinformation 4, no 1: 75-87.

Congalton, R.G. and K. Green. 2008. Assessing the accuracy of remotely sensed data: Principles and practices. Boca Raton, Florida, United States: CRC Press.

Crist, E.P.K., R.J. 1986. The tasseled cap de-mystified. Photogrammetric Engineering and Remote Sensing 52: 81-86.

Dabboor, M., S. Howell, M. Shokr and J. Yackel. 2014. The jeffries-matusita distance for the case of complex wishart distribution as a separability criterion for fully polarimetric sar data. International Journal of Remote Sensing 35, no 19: 6859-73.

Ding, Y.L., K. Zhao, X.M. Zheng and T. Jiang. 2014. Temporal dynamics of spatial heterogeneity over cropland quantified by time-series ndvi, near infrared and red reflectance of landsat 8 oli imagery. International Journal of Applied Earth Observation and Geoinformation 30: 139-45.

Farewell, T.S., V.T. Farewell and D.M. Farewell. 2013. Assessing measures of map value for thematic maps with sparse data. International Journal of Remote Sensing 34, no 8: 2655-71.

Fletcher, R. 1981. Practical methods of optimization. Volume 2: Constrained optimization (v. 2). New York: John Wiley \& Sons Ltd.

Goodwin, N.R. and L.J. Collett. 2014. Development of an automated method for mapping fire history captured in landsat tm and etm plus time series across queensland, australia. Remote Sensing of Environment 148: 206-21.

Hartfield, K., S. Marsh, C. Kirk and Y. Carriere. 2013. Contemporary and historical classification of crop types in arizona. International Journal of Remote Sensing 34, no 17: 6024-36.

Huang, G.-B., Q.-Y. Zhu and C.-K. Siew. 2004. Extreme learning machine: A new learning scheme of feedforward neural networks. In International Joint Conference on Neural Networks (IJCNN2004), 985--90. Budapest, Hungary.

Huang, G.B., H.M. Zhou, X.J. Ding and R. Zhang. 2012. Extreme learning machine for regression and multiclass classification. Ieee Transactions on Systems Man and Cybernetics Part B-Cybernetics 42, no 2: 513-29.

Langner, A., Y. Hirata, H. Saito, H. Sokh, C. Leng, C. Pak and R. Rasi. 2014. Spectral normalization of spot 4 data to adjust for changing leaf phenology within seasonal forests in cambodia. Remote Sensing of Environment 143: 122-30.

Laurin, G., F. Del Frate, L. Pasolli, C. Notarnicola, L. Guerriero and R. Valentini. 2013. Discrimination of vegetation types in alpine sites with alos palsar-, radarsat-2-, and lidar-derived information. International Journal of Remote Sensing 34, no 19: 6898-913.

Matthew, M.W., S.M. Adler-Golden, A. Berk, S.C. Richtsmeier, R.Y. Levine, L.S. Bernstein, P.K. Acharya, G.P. Anderson, G.W. Felde, M.L. Hoke, A.J. Ratkowski, H.-H.K. Burke, R.D. Kaiser and D.P. Miller. 2000. Status of atmospheric correction using a modtran4based algorithm. Paper presentat at the SPIE 4049, Algorithms for Multispectral, Hyperspectral, and Ultraspectral Imagery VI, in Orlando, FL. 
Mcnemar, Q. 1947. Note on the sampling error of the difference between correlated proportions or percentages. Psychometrika 12, no 2: 153-57.

Pal, M., A.E. Maxwell and T.A. Warner. 2013. Kernel-based extreme learning machine for remote-sensing image classification. Remote Sensing Letters 4, no 9: 853-62.

Pontius, R. and M. Millones. 2011. Death to kappa: Birth of quantity disagreement and allocation disagreement for accuracy assessment. International Journal of Remote Sensing 32, no 15: 4407-29.

Puertas, O., A. Brenning and F. Meza. 2013. Balancing misclassification errors of land cover classification maps using support vector machines and landsat imagery in the maipo river basin (central chile, 1975-2010). Remote Sensing of Environment 137: 112-23.

Rcoreteam. R: A language and environment for statistical computing.

Richards, J.A. 1999. Remote sensing digital image analysis: Berlin: Springer-Verlag.

Sarker, L. and J. Nichol. 2011. Improved forest biomass estimates using alos avnir-2 texture indices. Remote Sensing of Environment 115, no 4: 968-77.

Sonobe, R., H. Tani and X. Wang. 2014a. Short communication. Detection of ambrosia beetles using a pan-sharpened image generated from ALOS/AVNIR-2 and ALOS/PRISM imagery. Forest Systems 23, no 1: 178-82.

Sonobe, R., H. Tani, X. Wang, N. Kobayashi and H. Shimamura. 2014b. Parameter tuning in the support vector machine and random forest and their performances in cross- and same-year crop classification using TerraSAR-X. International Journal of Remote Sensing 35, no 23: 7898-909.

Sonobe, R., H. Tani, X. Wang, N. Kobayashi and H. Shimamura. 2014c. Discrimination of crop types with TerraSAR-X-derived information. Physics and Chemistry of the Earth, http://dx.doi.org/10.1016/j.pce.2014.11.001

Therneau, T., B. Atkinson and B. Riple. Package 'rpart'. http://cran.rproject.org/web/packages/rpart/rpart.pdf.

Varshney, A., M.K. Arora and J.K. Ghosh. 2012. Median change vector analysis algorithm for land-use land-cover change detection from remote-sensing data. Remote Sensing Letters 3, no 7: 605-14.

Yuan, L., J.C. Zhang, Y.Y. Shi, C.W. Nie, L.G. Wei and J.H. Wang. 2014. Damage mapping of powdery mildew in winter wheat with high-resolution satellite image. Remote Sensing 6, no 5: 3611-23. 
Table 1. Cultivation calendar for the crops in this study area.

Table 2. Crop type and number of fields.

Table 3. Accuracy results for CART and KELM.

Table 4. Z-test results.

Figure 1 . The study area.

Figure 2. J-M distances for OLI data. The thick lines show that the J-M distance values are greater than 1.7, in at least one band, and the dotted lines represent below 1.0 in every band. Bands 1 to 7 are the reflectance for OLI data and bands 8 to 13 are the KT data (brightness, greenness, wetness, haziness, fifth and sixth).

Figure 3. Decision tree by CART using the training data. The tree keeps splitting the branch using (a) the reflectance (\%) of the bands, (b) the KT data, and (c) the reflectance of the bands and the KT data. 
Table 1. Cultivation calendar for the crops in this study area.

\begin{tabular}{|c|c|c|c|c|c|c|c|c|c|c|}
\hline & & April & May & June & July & August & September & October & November & December \\
\hline \multicolumn{2}{|l|}{ Beet } & & & & & & & & & \\
\hline \multicolumn{2}{|l|}{ Corn } & & & & & $E$ & $\Rightarrow$ & & & \\
\hline \multirow{2}{*}{\multicolumn{2}{|c|}{ Winter wheat }} & & & & & & SUMU & & & \\
\hline & & & & & & ב & & & & \\
\hline \multirow[t]{2}{*}{ Beans } & Azuki & & $\mathbb{N}$ & & & & 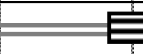 & 要 & & \\
\hline & Soy & & M & & & & & & $\equiv$ & \\
\hline Potato & & & $\$$ & & & & & & & \\
\hline
\end{tabular}

plantation/transplanting

E ripeness/harvesting 
Table 2. Crop type and number of fields.

\begin{tabular}{ccc}
\hline Crop type & \multicolumn{2}{c}{ No. of fields } \\
& Training data & Test data \\
\hline Beans & 80 & 320 \\
Beets & 68 & 272 \\
Grassland & 65 & 257 \\
Maize & 50 & 200 \\
Potatoes & 50 & 199 \\
Wheat & 180 & 726 \\
\hline
\end{tabular}


Table 3. Accuracy results for CART and KELM.

\begin{tabular}{|c|c|c|c|c|c|c|}
\hline \multirow{2}{*}{$\begin{array}{r}\begin{array}{r}\text { Data } \\
\text { Class }\end{array} \text { Algorithm } \\
\end{array}$} & \multicolumn{2}{|c|}{ Reflectance } & \multicolumn{2}{|c|}{ KT data } & \multicolumn{2}{|c|}{$\begin{array}{c}\text { Reflectance } \\
\text { + KT data }\end{array}$} \\
\hline & CART & KELM & CART & KELM & CART & KELM \\
\hline \multicolumn{7}{|l|}{ Producer's accuracy } \\
\hline Beans & 0.753 & 0.919 & 0.781 & 0.916 & 0.916 & 0.919 \\
\hline Beets & 0.926 & 0.930 & 0.868 & 0.934 & 0.926 & 0.923 \\
\hline Grassland & 0.704 & 0.798 & 0.751 & 0.790 & 0.716 & 0.794 \\
\hline Maize & 0.745 & 0.725 & 0.510 & 0.725 & 0.610 & 0.720 \\
\hline Potatoes & 0.899 & 0.899 & 0.834 & 0.899 & 0.905 & 0.894 \\
\hline Wheat & 0.908 & 0.968 & 0.909 & 0.966 & 0.908 & 0.961 \\
\hline \multicolumn{7}{|l|}{ User's accuracy } \\
\hline Beans & 0.715 & 0.788 & 0.639 & 0.788 & 0.701 & 0.776 \\
\hline Beets & 0.834 & 0.907 & 0.764 & 0.910 & 0.834 & 0.903 \\
\hline Grassland & 0.823 & 0.949 & 0.828 & 0.931 & 0.939 & 0.944 \\
\hline Maize & 0.608 & 0.780 & 0.590 & 0.784 & 0.667 & 0.778 \\
\hline Potatoes & 0.873 & 0.873 & 0.917 & 0.869 & 0.857 & 0.873 \\
\hline Wheat & 0.991 & 0.983 & 0.961 & 0.982 & 0.991 & 0.980 \\
\hline Overall accuracy & 0.841 & 0.901 & 0.814 & 0.899 & 0.856 & 0.896 \\
\hline $\begin{array}{l}\text { Allocation } \\
\text { disagreement }\end{array}$ & 10.892 & 6.535 & 13.121 & 6.738 & 7.345 & 6.839 \\
\hline Quantity disagreement & 4.965 & 3.343 & 5.471 & 3.343 & 7.042 & 3.546 \\
\hline
\end{tabular}


Table 4. Z-test results.

\begin{tabular}{|c|c|c|c|c|c|c|c|}
\hline \multirow{2}{*}{\multicolumn{2}{|c|}{ classification }} & \multicolumn{2}{|c|}{ (1) } & \multicolumn{2}{|c|}{ (2) } & \multicolumn{2}{|c|}{ (3) } \\
\hline & & CART & KELM & CART & KELM & CART & KELM \\
\hline \multirow{2}{*}{ (1) } & CART & $\mathrm{x}$ & 5.75 & 2.49 & 5.53 & 1.31 & 5.21 \\
\hline & KELM & & $\mathrm{x}$ & 8.25 & 0.22 & 4.44 & 0.54 \\
\hline \multirow{2}{*}{ (2) } & CART & & & $\mathrm{x}$ & 8.03 & 3.80 & 7.70 \\
\hline & KELM & & & & $\mathrm{x}$ & 4.22 & 0.32 \\
\hline \multirow{2}{*}{ (3) } & CART & & & & & $\mathrm{x}$ & 3.90 \\
\hline & KELM & & & & & & $\mathrm{x}$ \\
\hline
\end{tabular}




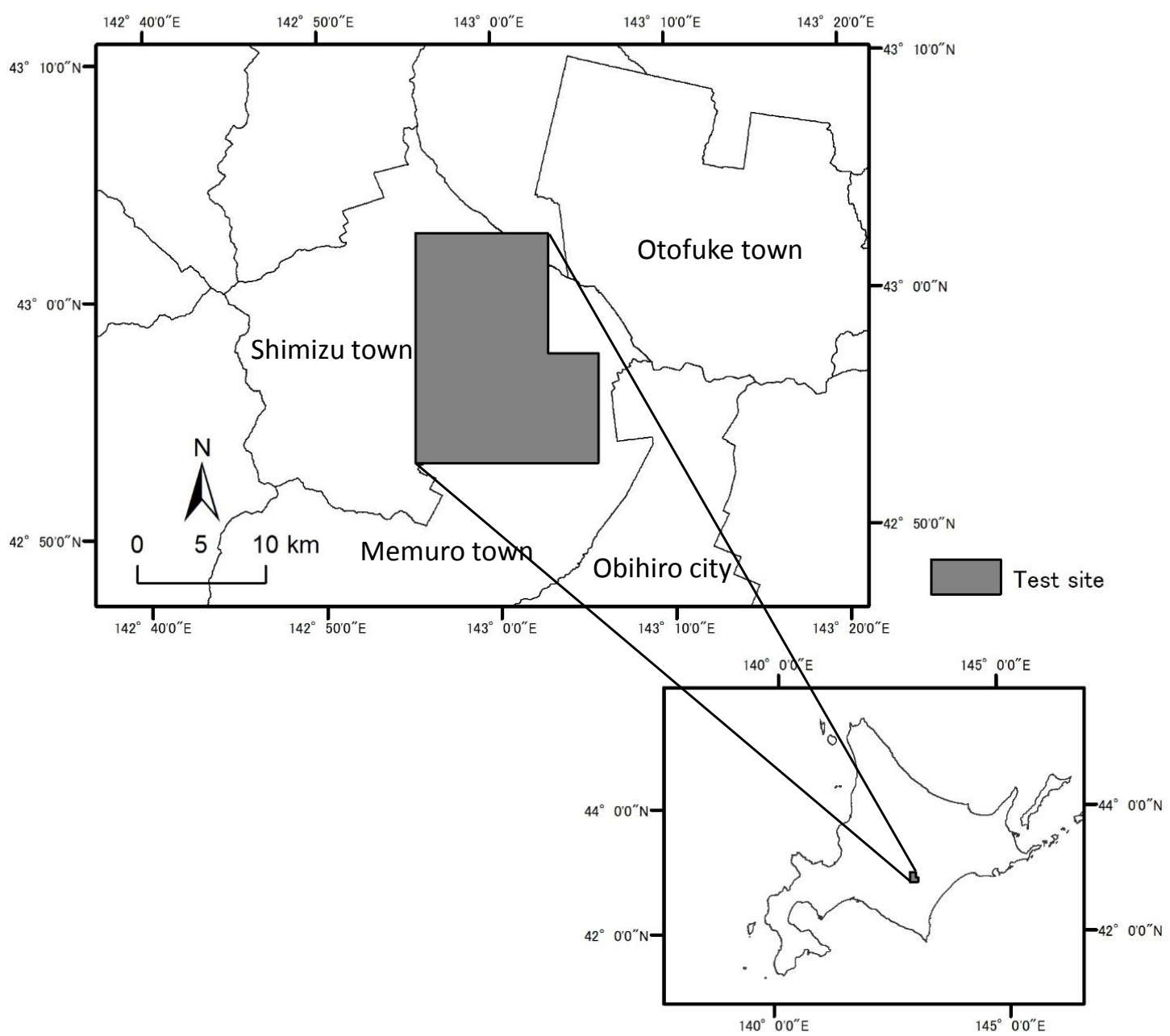

Figure 1. The study area. 
(a)

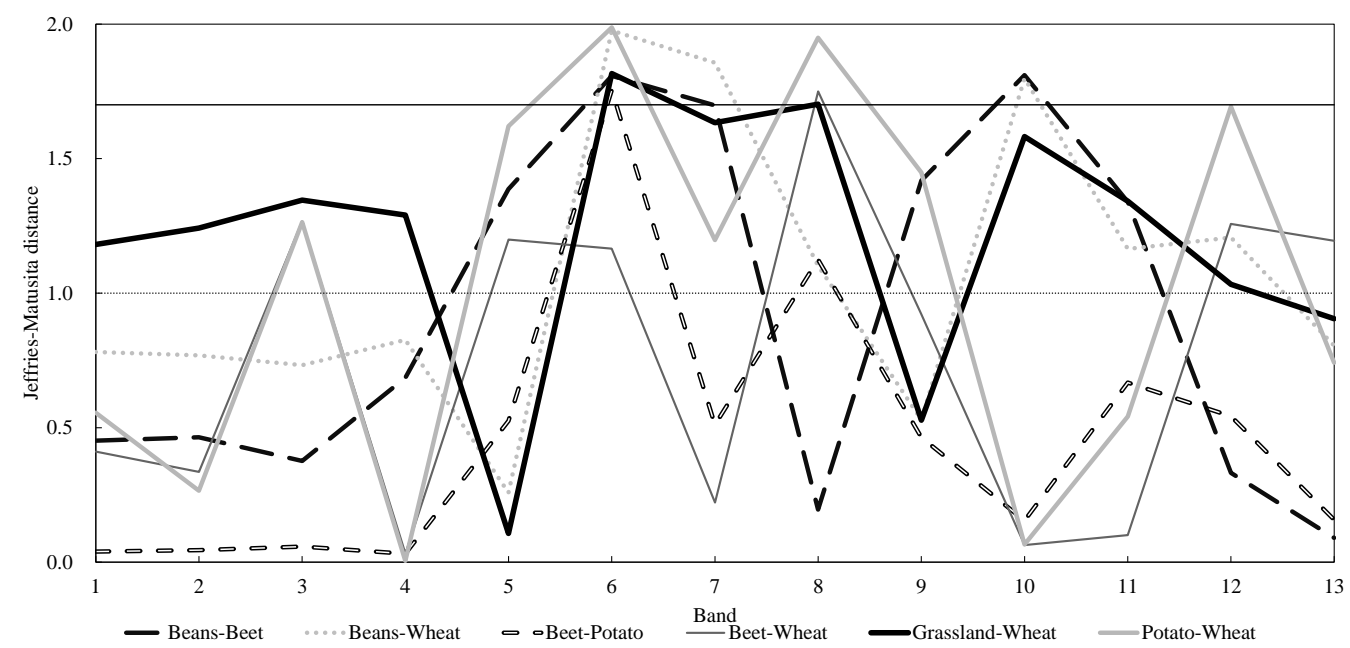

(b)

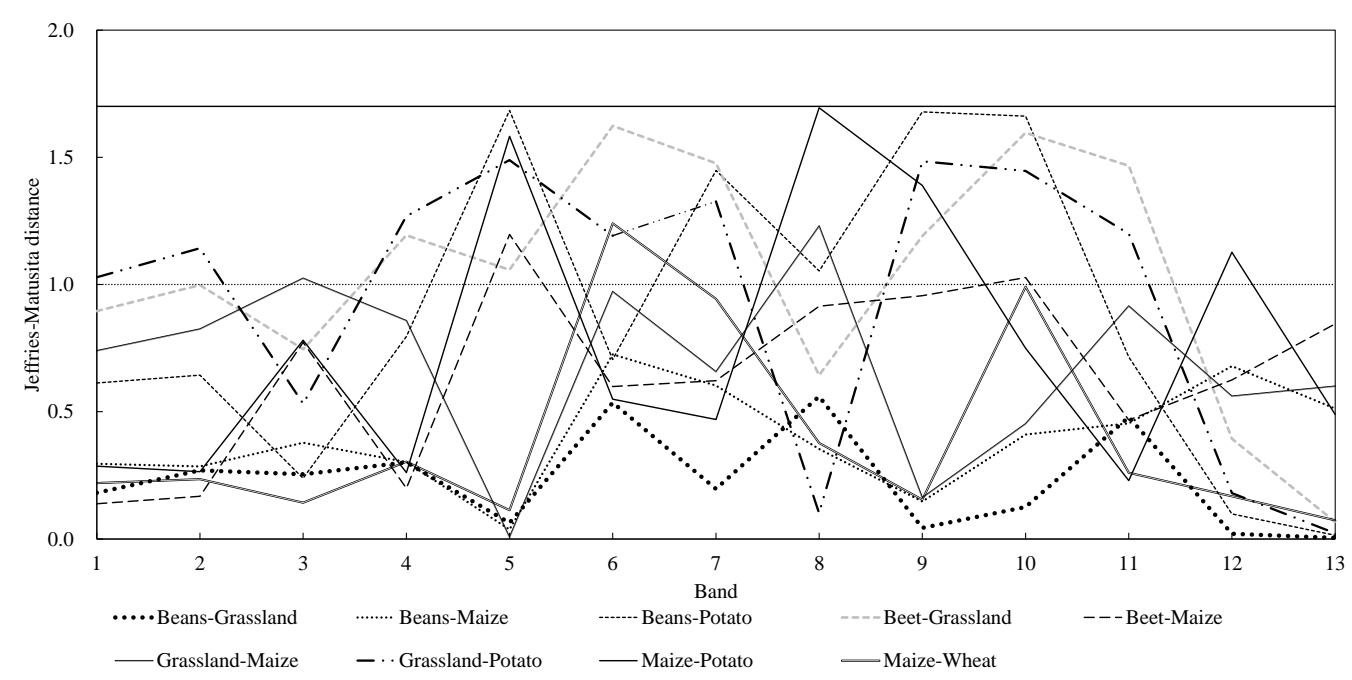

Figure 2. J-M distances for OLI data. Bands 1 to 7 are the reflectance for OLI data and bands 8 to 13 are the KT data (brightness, greenness, wetness, haziness, fifth and sixth). (a) Curves with J-M distances values are greater than 1.7 in at least one band, (b) curves with J-M distances values are less than 1.0 in every band. 
(a) Reflectance

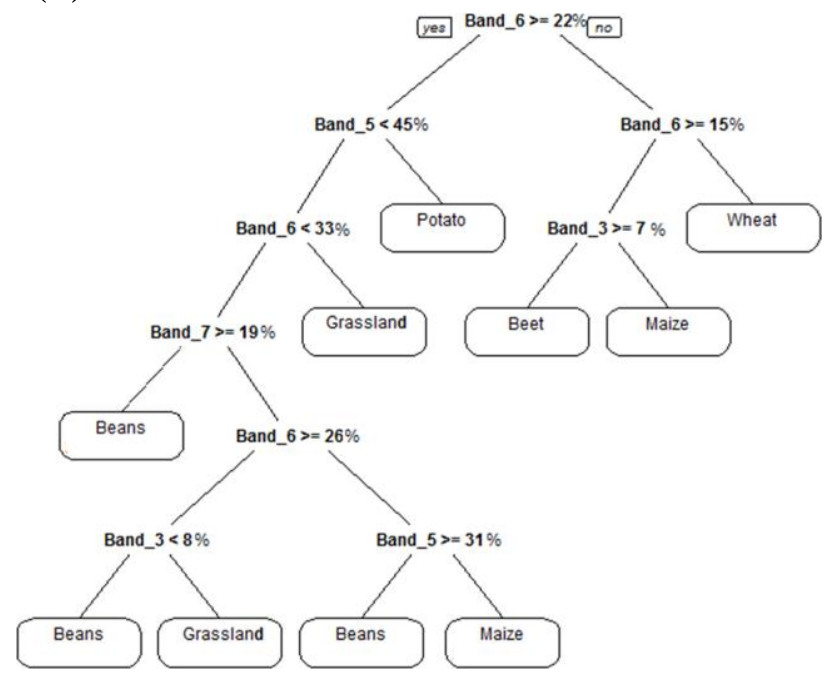

(b) Kauth Thomas data

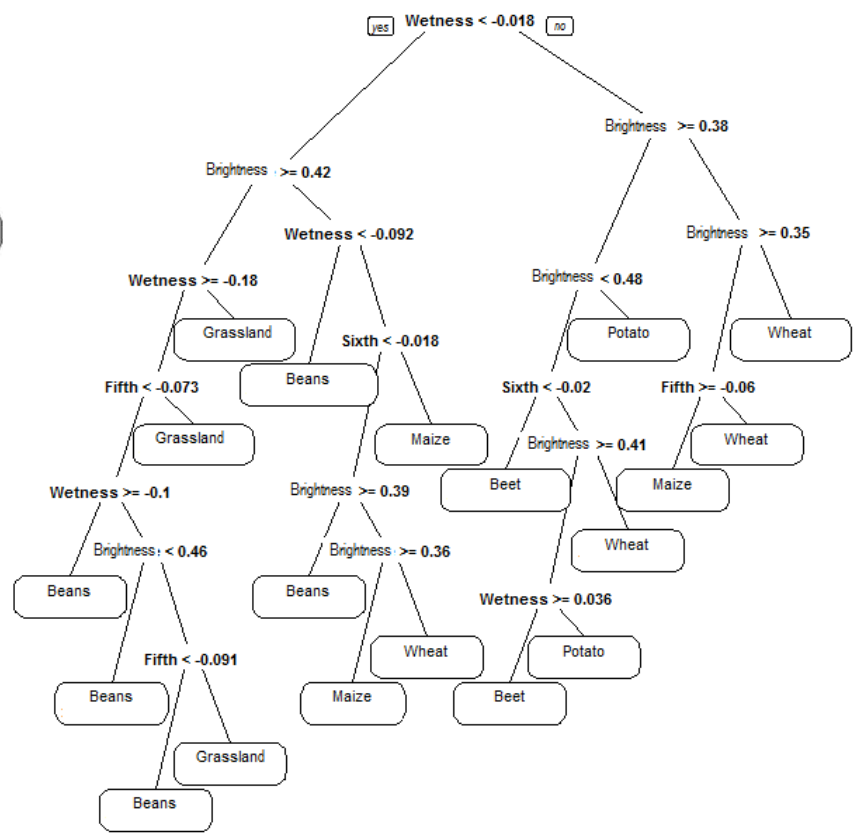

(c) Reflectance + Kauth Thomas data

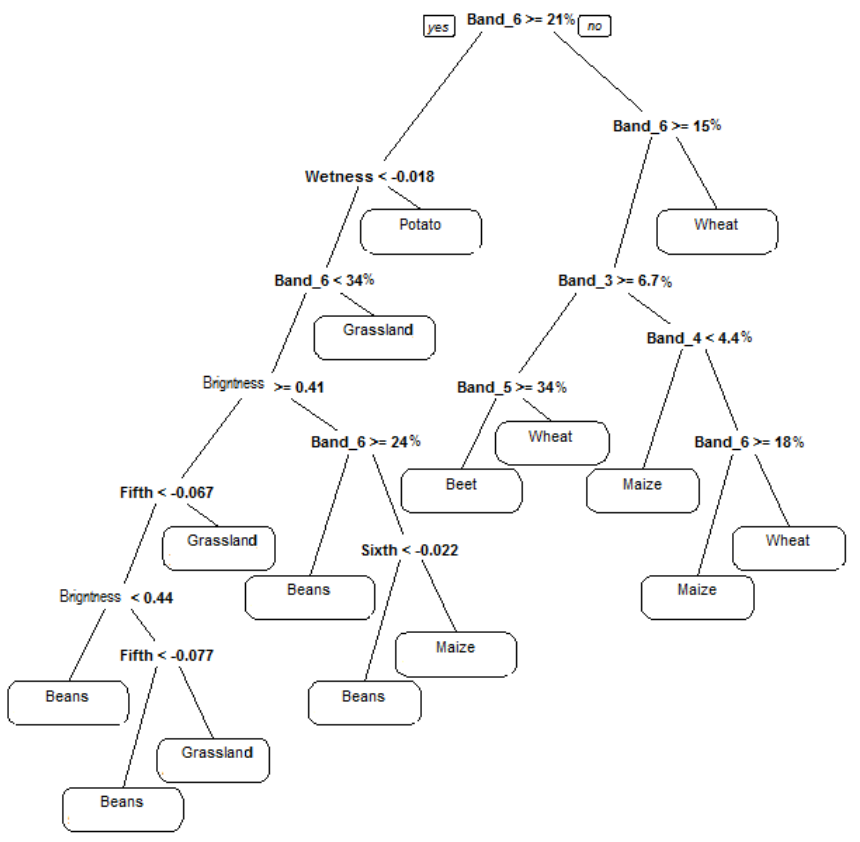

Figure 3. Decision tree by CART using the training data. The tree keeps splitting the branch using (a) the reflectance (\%) of the bands, (b) the KT data, and (c) the reflectance of the bands and the KT data. 